\title{
An optimized multi agent-based modeling of smart rescue operation
}

\author{
S. Azimi ${ }^{1}$, M. R. Delavar ${ }^{2}$, A. Rajabifard ${ }^{3}$ \\ ${ }^{1}$ GIS Dept., School of Surveying and Geospatial Engineering., College of Engineering, University of Tehran, Tehran, Iran- \\ sanazazimi@ut.ac.ir \\ ${ }^{2}$ Center of Excellence in Geomatic Engineering in Disaster Management, School of Surveying and Geospatial Engineering., College \\ of Engineering, University of Tehran, Tehran, Iran-mdelavar@ut.ac.ir \\ ${ }^{3}$ Department of Infrastructure Engineering, Centre for Spatial Data Infrastructures and Land Administration, the University of \\ Melbourne, Melbourne, VIC, Australia-abbas.r@unimelb.edu.au
}

\section{Commission IV, WG IV/3}

KEY WORDS: Multi-agent based modeling, Resource allocation, Multiplicatively Weighted Network Voronoi Diagram, Particle Swarm Optimization, Smart rescue operation, Smart city

\begin{abstract}
:
After the incidence of a disaster, a high demand for first-aid and a huge number of injured will emerge at the affected areas. In this paper, the optimum allocation of the medical assistance to the injured according to a multi-criteria decision making is performed by Multiplicatively Weighted Network Voronoi Diagram (MWNVD). For consideration of the allocation of the injured in the affected area to the appropriate hospitals using the MWNVD and decreasing the gap between the estimated and expected population in the MWNVDs, Particle Swarm Optimization (PSO) is applied to the MWNVDs.

This paper proposes a multi agent-based modeling for incorporating the allocation of the medical supplies to the injured according to the generated Voronoi Diagrams of the PSO-MWNVD, wayfinding of emergency vehicles based on the minimum travel distance and time as well as using smart city facilities to expedite the rescue operation. In the proposed model, considering the priority of the injured for receiving the medical assistance, information transfer about the condition of the injured to the hospitals prior to ambulance arrival for providing appropriate treatment, updating of emergency vehicles route based on the blocked streets and etc. are optimized.

The partial difference between the estimated and expected population for receiving the medical assistance in MWNVDs is computed as $37 \%$, while the PSO-MWNVD decreased the mentioned difference to $6 \%$. The relief operation time in the proposed model compared to another multi-agent rescue operation model, which uses MWNVD and does not have some facilities of the proposed
\end{abstract} model, is improved.

\section{INTRODUCTION}

Disasters can occur in anytime and anyplace. The high density of the population, buildings, and infrastructure in cities cause them vulnerable to disasters (Zhuang and Bier, 2007, Li et al., 2011). Tabas, Rudbar and Bam earthquakes are the worst and deadliest earthquake disasters in the last 100 years in Iran (Ibrion et al., 2015). During the 208 natural disasters from 1900-2015 in Iran, 156332 have been died and many people have been affected (Ghomian and Yousefian, 2017). Providing the quick assistance to the wounded is a significant factor for increasing their survival probability.

There is a major need to the better allocation and the schedule of the existing medical assistances to provide the best feasible opportunities for survival, decreasing the loss of life and increasing the performance of the relief operation (Bostick et al., 2008). Therefore, the optimum allocation of ambulances to the emergency victims as fast as possible is essential where optimum routing of the ambulances to the emergency victims' locations is important at the same time. The optimum allocation of the victims to the appropriate emergency centers would require consideration of traffic conditions for transferring of the medical assistance to the wounded locations. Reducing the travel time of the ambulances to the emergency victims is one way to decrease the emergency vehicle response time (Billhardt et al., 2014).

Medical resource assignment has not been much considered in disaster management (Fiedrich et al., 2000, Gong and Batta, 2007).

There has been few research that explicitly study patients allocation problems to their most appropriate hospitals for receiving the medical assistance. In most of the studies, allocation of the nearest available ambulances to the emergency victims' location is performed. Bandara et al. (2014) showed that dispatching the nearest vehicle has not been applied in an optimum manner.

In some studies, the priority of the victims have been analyzed with regard to the severity of their injuries in order to receive the first aid (Bandara et al., 2014, Andersson and Värbrand, 2007, López et al., 2008)

In some researches, the response time for emergency medical services is analyzed. Haghani et al. (2003) proposed an optimization model for developing the flexible dispatching of the medical services by considering the ambulances available in the real-time.

On the other hand, the Voronoi Diagram (VD) can be used to determine the service area for urban facilities. Karimi et al. (2009) investigated the Ordinary Voronoi Diagram and multiplicatively weighted Voronoi Diagram for determining the space allocation of the educational centers.

Search and rescue operation is a good application for agentbased modeling. Because the agent-based modeling is a natural way to model relief groups during an emergency situation. According to Rusell and Norvig (2003), the agent perceives its environment through sensors and acts upon them through its effectors. Multi-Agent Systems (MAS) are composed of multiple agents interacting with each other and with objects in a single simulation environment, for complying a common goal (Roozemond, 2001). In some studies, the issue of the multiagent rescue operation is concerned. Chavoshi et al. (2008) proposed a novel agent-based model of wayfinding for rescue operation based on landmarks remained after the earthquake. 
Wang et al. (2014) proposed a multi-agent cooperative coverage control model for the resource assignment to provide a distributed convergent approach to the ambulance position. The Voronoi set for each ambulance was determined based on keeping track of its neighbours. As Hawe et al. (2015) have indicated agent-based modeling of the best allocation of resources for a hypothetical two-site incident in order to minimize transportation time of the injured to the appropriate hospital was performed. Wang and Zlatanova (2016) proposed a multi-agent system for navigation of the first responders to emergency situations due to moving obstacles such as fires, plumes, and floods.

Smart health and transportation facilities can expedite the rescue operation. In the smart city, the real-time routing of the vehicles by considering traffic flow information, street status and traffic incidents are performed that can assist decreasing the emergency vehicle response time ( $\mathrm{Li}$ et al., 2013).

The ambulances during their tasks have strengthened their communication with the related hospitals. On the other hand, ambulances transfer information about the condition of the injured to the hospitals prior to their arrival in order to provide appropriate treatment which can improve the survival probability of the patients. Also, the ambulance can get patient medical reports in real time (Alami-Kamouri et al., 2017).

There have been a number of studies on the rescue operations in the smart city. Billhardt et al. (2014) proposed a dynamic coordination model for ambulances fleet that combines ambulances redeployment mechanism with optimal assignment of ambulances to victim locations at each moment. Hirokawa and Osaragi (2016) investigated the accessibility of firefighters to the location of fire immediately after an earthquake by $\mathrm{A}^{*}$ algorithm. When volunteers gathered information on street blockage, the arrival time of the firefighters to the location of fire have been reduced. Alami-Kamouri et al. (2017) presented a mobile agent-based model in order to transfer the information on patient conditions from smart ambulance to a hospital and getting a patient medical report from the hospital to the smart ambulance in real time.

In this paper, we are faced with the situation how to expedite the allocation of medical assistance to the emergency victims after some disasters or accidents, based on the facilities of smart city and modeling of the multi-agent system.

The hypothesis of this paper is that optimum allocation of the medical services to the emergency victims' locations according to the priority of emergency victims, optimum wayfinding of ambulances by considering the blockage of streets and the smart city facilities can accelerate the rescue operation.

In this paper, the coordination among the agents in the model for ambulance fleets that provide the allocation of ambulances to the injured locations based on particle swarm optimizationMultiplicatively Weighted Network Voronoi Diagram (PSOMWNVD) is proposed. In the proposed multi agent-based model, the urban street network, population density, and the ambulances of the emergency centers, optimum wayfinding of emergency vehicles to the injured location by considering travel distance, travel time, the street blockage and smart city facilities to improve the rescue operation are considered. The coordination center, emergency centers, ambulances, fire stations, fire engines and the wounded are modeled as agents. In the proposed model it is assumed that the wounded delivered their locations by mobile Global Positioning System (GPS) and their initial symptoms to the coordination center agent.

The allocated Voronoi Diagram is determined for each emergency center. The number of ambulances are considered as the weight of the emergency centers. The Bi-label Dijkstra algorithm is used for wayfinding of ambulances in order to minimize travel length and travel time of the ambulances in the street network. Two types of multi-agent rescue operation simulations are implemented and their results are compared. In the first type of the multi-agent rescue operation, the MWNVD is determined for each emergency center. The ambulances from proper emergency center based on the MWNVD that include the location of the wounded, according to the priority of the wounded and the ambulance numbers which are dispatched from the emergency center to the wounded sites are considered. This process is performed simultaneously for all the injured in different Voronoi Diagrams. In the case that the ambulance capacity of each emergency center became zero, the response time to new demand is delayed until the return of the first ambulance to the emergency center. In the second type of the multi-agent rescue operation, the PSO-MWNVD and the optimum weight for each of the emergency centers are computed. The ambulance from proper emergency center based on PSO-MWNVD according to the severity level of the wounded and the optimum ambulance numbers of the emergency center is transferred to the wounded site. The ambulances' route are updated according to blocked streets, if it is possible. Also, the ambulances are able to transfer information about the condition of the injured to the hospitals prior to their arrival in order to provide appropriate treatment. In case that all the ambulances of each emergency center are already dispatched, PSO-MWNVD for other emergency centers are activated and the rescue process is continued. Finally, these two types of multi-agent rescue operation models are compared according to the optimum generated Voronoi Diagrams, the arrival time of ambulances to the patient location and the duration time of ambulance's mission.

The outline of the rest of the paper is as follows. Section 2 provides the fundamental concepts for the proposed multi agent-based model. Section 3 explains the methodology of the allocation of the wounded to the emergency centers, the wayfinding of the ambulances in the disaster situation and the facilities of the smart city for accelerating the rescue operation in the multi agent-based model. Section 4 explains implementation and results of the proposed multi-agent rescue operation. Finally, Section 5 presents conclusions and directions for future research.

\section{FUNDAMENTAL CONCEPTS}

\subsection{Voronoi Diagram (VD)}

The Voronoi Diagram (VD) represents the partitioning of the given space into the regions based on a set of generators such that for each generator a corresponding region is determined. In our application, the two-dimensional VD is considered. Network Voronoi Diagram (NVD) is the street network based VD that is utilized for allocating service areas to each emergency centers (Okabe et al., 2000). The NVD partitions the nodes and the arcs of the network. In principle there are three types of NVDs including the Node Network Voronoi Diagram, the Arc Network Voronoi Diagram and the Area Network Voronoi Diagram (Okabe et al., 2000).

The Bi-Label Dijkstra shortest path algorithm is used for the construction of the NVD.

\subsubsection{Multiplicatively Weighted Network Voronoi Diagram}

In Weighted Network Voronoi Diagram (WNVD), a weight is considered for all points. The weight of each generator is 
dissimilar in a Multiplicatively Weighted Network Voronoi Diagram (MWNVD).

By MWNVD, the demand nodes are assigned to the nearest emergency center which may cause an overloaded or underloaded problem in many VDs. The iteration process is applied to the MWNVDs in order to reduce the difference between the expected population in each MWNVD for using the services and the estimated population in the same MWNVD.

\subsubsection{Particle swarm optimization - Multiplicatively} Weighted Network Voronoi Diagram

Particle Swarm Optimization algorithms (PSO) (Yang, 2010, Santosa, 2006) are based on the movement and swarm behaviour of birds flocking or fish schooling in nature, originally accredited to Eberhart and Kennedy (1995). PSO is a metaheuristic and stochastic optimization algorithm that searches an objective spaces for attaining the best solution. Two major components of the movement of a swarming particle are a stochastic component and a deterministic component. Each particle is attracted toward the best position of their neighbourhood called global best (gBest) and its own best location called local best (lBest) in history. The particle position in the search space changes with respect to the social psychological behaviour of other particles and mimics the success of other particles. The position of particles is affected by velocity of the particles (Bai, 2010, Engelbrecht, 2007, Santosa, 2006). The velocity and position of the particles at are computing using Eq. 1 and Eq. 2, respectively (Santosa, 2006, Engelbrecht, 2006):

$$
\begin{aligned}
& v_{i}(t+1)=a v_{i}(t)+c_{1} r_{1}\left[\operatorname{lBest}(t)-x_{i}(t)\right]+ \\
& c_{2} r_{2}\left[g \operatorname{Best}(t)-x_{i}(t)\right] \\
& x_{i}(t+1)=x_{i}(t)+v_{i}(t+1)
\end{aligned}
$$

where $x_{i}(0) \sim u\left(x_{\min }, x_{\max }\right)$ and $a$ promotes local exploitation. $c_{1}$ and $c_{2}$ coefficients are learning rates for individual influence (cognitive component) and social influence (social component) that usually considered as $2 . r_{1 j}$ and $r_{2 j}$ are the random vectors in the range $[0,1]$. The $x_{i}(t+1)$ denotes the position of particle $i$ in time step $t+1, t$ is discrete time steps and $v_{i}(t+1)$ is the velocity of the particle $i$ in the time step $t+1$.

The procedure of PSO can be summarized as the following steps.

At first, the size of the group of particles $(M)$, the coefficients of $\epsilon_{1}, \epsilon_{2}$ and $a$ are specified. Then a random swarm is generated. After selecting the objective function, the value of the objective function is computed for each particle. The initial velocity of all the particles are assumed to be zero. All of the particles move towards the optimum point with a velocity. In each iteration, the lBest for each particle with the lowest value of an objective function in the minimization case, gBest, and the best value for all the particles, the velocity and position of each particle are calculated.

Also, the aim of this optimization is to find the gBest among all the current local best solutions after a certain number of iterations $\left(N_{\max }\right)$ or until the objective improves. This process is repeated until the termination criteria is met (Rini et al., 2011). In Particle Swarm Optimization-Multiplicatively Weighted Network Voronoi Diagram (PSO-MWNVD), the PSO algorithm is applied to MWNVD to determine optimum service areas for each emergency center.
In this paper, the number of ambulances of each emergency center is regarded as an emergency center weight. In the PSOMWNVD algorithm, the initial position of each particle within the search space is determined by the initial weight of the emergency centers. The weight of the emergency centers is updated for each particle per iteration using Eq. 2. The process of the PSO-MWNVD continues until the difference between the demand and supply in the MWNVDs become minimum.

In the PSO-MWNVD algorithm, the mean proportional absolute error $(E r)$ is regarded as the termination criteria to determine optimum service area for each emergency center. The error metric, $E r$, can be used for assessing partitioning method which is presented in Eq. 3 (Reitsma et al., 2007, Wood, 1974).

$$
\mathrm{Er}=\frac{1}{\mathrm{~N}} \sum_{\mathrm{i}=1}^{\mathrm{N}} \frac{\mathrm{P}_{\mathrm{j}}-\mathrm{p}_{\mathrm{i}, \mathrm{j}}}{\mathrm{P}_{\mathrm{j}}}
$$

where the $p_{i, j}$ is the estimated population of the service areas of emergency center $j$ in iteration $i$ as proportion of the total population. $p_{i j j}$ is get from the sum of the population in service areas of emergency center $j . P_{j}$ is the expected population for receiving the medical assistance in service areas of emergency center $j$ as proportion of the total population. $N$ is the total number of iterations, respectively.

The performance of Bi-Label Dijkstra shortest path algorithm for wayfinding the ambulances to the wounded location is explained in the next section.

\subsection{Bi-Label Dijkstra algorithm}

In this paper, the Bi-label Dijkstra shortest path algorithm is used for the construction of the MWNVD. Each node in the network is assigned to singular emergency center by calculating the Bi-label Dijkstra shortest path algorithm based on the minimum travel time and the travel length from the node to the emergency center in the street network.

The bi-objective shortest path problem is a development of the classical shortest path problem that is related to the class of multi-objective optimization problems. In the specific network, the Bi-objective Shortest Path Problem uses for specifying the set of non-dominated paths between nodes by optimizing the two objective functions (Ticha et al., 2017, Serafini, 1987).

In the Bi-label Dijkstra algorithm, each label represents a sub path from a source node $v_{0}$ to a certain node $u \in V$ that is represented as $L=\left(u_{s} d(L), t(L)\right)$ where $u$ is the ending node of the sub path $L$. In addition, $d(L)$ and $t(L)$ represent the total distance and the total travel time associated with the sub path, respectively.

In this paper, Lexicographic order is used for comparing the labels to determine the optimum path. A vector $a=\left(a_{1}, a_{2}\right)$ is lexicographically smaller than a vector $b=\left(b_{1}, b_{2}\right)$, denoted by $a<b$ if either $a_{1}<b_{1}$ or both $a_{1}=b_{1}$ and $a_{2}<b_{2}$. A path $p_{1}$ lexicographically smaller than path $p_{2}$ if and only if $\left(t\left(p_{1}\right), d\left(p_{1}\right)\right)<_{\text {Tex }}\left(t\left(p_{2}\right), d\left(p_{2}\right)\right)$. Similarly a label $L_{1}<_{\text {iex }} L_{2}$ if and only if $\left(t\left(L_{1}\right), d\left(L_{1}\right)\right)<_{\text {lex }}\left(t\left(L_{2}\right), d\left(L_{2}\right)\right)$ (Pyrga et al., 2008).

\subsection{Smart transportation and Smart health}

In the smart city (Li et al., 2013, Bakıc1 et al., 2013), better and safer living conditions for citizens, including lack of restrictions on transportation and medical care, city administration, crisis management and so on are established. 
The traditional vehicle routing is limited to personal experience of drivers that often cause traffic congestion and inappropriate usage of certain road resources. In a smart city, the real-time routing of vehicles according to traffic density information, street condition, temperature and traffic accidents are recorded by sensors (Li et al., 2013).

Intelligent transportation systems (ITS) are a symbol of smart cities. ITS centers collect and reserve real-time road traffic data from various sources to reduce traffic congestion problems (Imawan et al., 2016).

To make closer a city to the smart city, improving the different areas that are part of the city such as the health sector is

necessary (Alami-Kamouri et al., 2017).

In disaster response situation, if information about condition of the wounded are announced to hospitals prior to their arrival, the hospital can prepare pre-arrival support to the wounded. So, the relevant actions about the appropriate treatment or operation can be performed in the hospitals. Also, the ambulance team can get additional information about special patients from the hospitals and perform proper actions. So, the ambulance team and the hospitals team can more adequately response, quickly provide services to the wounded and avoid delay in commencing patient treatment (Alami-Kamouri et al., 2017, Mustafa, 2013).

\section{METHODOLOGY}

After the occurrence of a natural disaster, the locations and initial symptoms of the wounded are delivered to the coordination center. The priority of the wounded according to their severity level is specified. The wounded with severe injuries more quickly receive the medical assistance. The service areas of emergency centers for delivering medical assistance to the wounded according to the population, supply, and the street network are determined by MWNVD and PSOMWNVD. The number of ambulances in each emergency center is considered as an emergency center weight. The Bi-label Dijkstra algorithm is applied for wayfinding of ambulances in order to minimize the ambulances travel time and the route length in the street network. In this study, the two types of rescue operations are analyzed.

Agent-based simulation (ABS) is able to provide a natural description of the rescue operation situations. The multi agentbased rescue operation for modeling the allocation of ambulances to the injured location, reallocation of ambulances and the facilities of the smart city in order to improve the relief operation for these two types of the rescue operation is performed.

As shown in Figure 1, the coordination center, the emergency centers, ambulances, fire stations, fire engines and the wounded are modeled as agents in Anylogic simulation software with specific attributes and behaviors. The wounded agent delivers help demand to the coordination center agent. The coordination center agent receives the wounded message, updates its memory and delivers the wounded message to the proper emergency center according to the VD. The emergency center agent receives the wounded message, updates its memory and assigns the task of providing medical assistance for the wounded to the ambulance agent. The ambulance agent is dispatched to the wounded location and communicates with the emergency center agent during its mission. Also, the ambulance agent will report the coordination center agent in case some streets are blocked and update its route accordingly. The fire station agent receives the blocked street message from the coordination center agent, updates its memory and assigns a task to fire engine agent. The fire engine agent is dispatched to the blocked streets and performs the relevant actions.

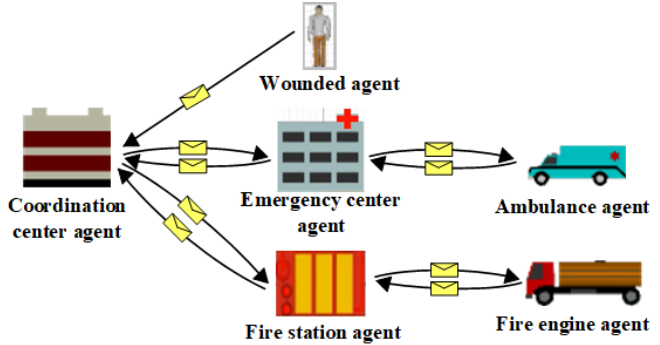

Figure 1. The communication of agents in the proposed model

The two types of the rescue operation were implemented in the multi agent-based simulation environment.

In the first type of the rescue operation, after determining the MWNVD for each emergency center, the proper emergency center according to the generated MWNVDs is allocated to the demand location. The emergency center due to the priority of the demand is delivered the ambulance to the demand location. The ambulance route is determined according to the goals of minimizing the route length and travel time at the beginning of its movement which is unable to update when faced with the blocked street. So, the ambulance will be delayed until the fire engine resolved the blockage of the street. The initial ambulance number of the emergency center is considered in the computation. In the case that ambulance capacity of each emergency center is fully employed, a new demand will not be delivered to the related emergency center until at least one of the ambulances of this emergency center completes its mission and returns to the emergency center. In this case, the wounded demand for receiving the first aid will be answered late and the injured survival probability will be reduced.

In the second type of the rescue operation, the PSO iterative mechanism in the MWNVD, decreases the difference between supply (the expected population lives in the region for using the services) and demand (the estimated population lives in the region) is performed. So, the optimum PSO-MWNVD and optimum ambulance number for each emergency center are determined. The ambulance from proper emergency center that is located in PSO-MWNVD that include the location of the wounded, according to the severity level of the wounded and the existing ambulances of the emergency center is dispatched to the wounded location. During the mission, the ambulance can benefit from the smart city facilities that are explained below. The optimum ambulance route is specified in order to minimize the route length and the travel time at the beginning of its movement. If the ambulance is faced with some blocked streets, the optimum route from the current location to the destination location is re-calculated, if it is possible. The ambulance on the way back from the injured location to the emergency center, is able to send the name and the current state (such as heart rate, body temperature, blood pressure and blood type) of the injured to the hospital. Figure 2 shows the list of some relevant information about the condition of the patients en route to the hospital. Figure 3 shows the two-way communication between the hospital and the ambulance. In the case that the ambulances of many emergency centers during the mission are fully employed, responding to the new demand is different form the first type of the multi-agent rescue operation. In this case, PSOMWNVD is re-calculated for the emergency centers that have ambulances without mission and the rescue operation process is continued. 


\begin{tabular}{|llll|}
\hline $\begin{array}{l}\text { Identification } \\
\text { Name: } \\
\text { SSN }^{1}:\end{array}$ & $\begin{array}{l}\text { Time: } \\
\text { Age: }\end{array}$ & $\begin{array}{l}\text { Date: } \\
\text { Gender: }\end{array}$ & $\begin{array}{l}\text { Chief complaint: } \\
\text { Phone number: }\end{array}$ \\
\hline $\begin{array}{l}\text { Vital Signs } \\
\text { Pulse Rate: }\end{array}$ & Blood Type: & $\begin{array}{l}\text { Right Pupil: } \\
\text { Left Pupil: }\end{array}$ & $\begin{array}{l}\text { Blood Pressure: } \\
\text { SPO2 }^{2}:\end{array}$ \\
\hline $\begin{array}{l}\text { Status } \\
\text { Perfusion: }\end{array}$ & Burns: & Skin Condition: & Wounds: \\
Mental State: & Bleeding: & Skin Color: & Fractures: \\
\hline
\end{tabular}

Figure 2. List of information about condition of the wounded sent by the ambulance to the hospital (Gao et al., 2006)

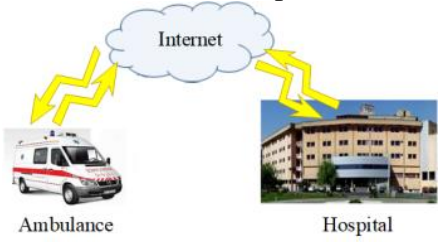

Figure 3. The two-way communication between the ambulance and the hospital

\section{IMPLEMENTATION}

The proposed multi-agent rescue operation is implemented in a part of municipal District 5 of Tehran, the capital of Iran. Figure 4 represents the study area that is limited to the Hakim highway from North, the Sanaye Havapeymayi Street and Sattari highway from West, the Lashkari highway from South and the Jenah highway from East. The topographic map of National Cartographic Center (NCC) at the scale of 1:2000 updated in 2003 and census data extracted from the demographic census at the scale of 1:2000 carried out in 2007 by Statistical Centre of Iran were used in this study. The emergency center part of the Sarem Specialist hospital, Ebne Sina hospital, Fatemeh Zahra health center and Payambaran hospital are located in municipal District 5 of Tehran that have 3, 4, 1, and 5 ambulances which are shown in Figure 4. The locations of emergency centers were acquired from municipality District 5 of Tehran. Also, the studied fire stations and population density of the study area are represented in Figure 4. The travel time of the vehicles on the urban street network of municipal District 5 of Tehran in 2017 is achieved from Municipality of Tehran Transport and Traffic Company.

In this paper, MWNVD and PSO-MWNVD allocation for the emergency center are investigated. The generated MWNVDs

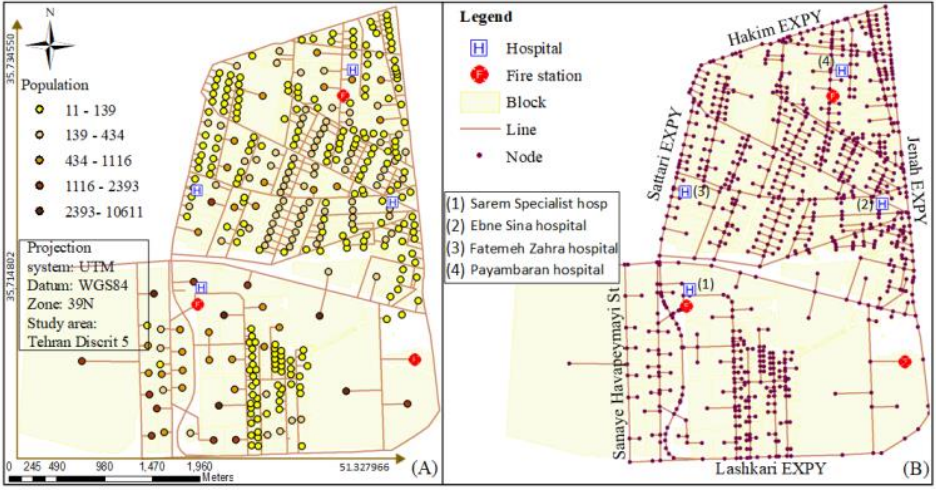

Figure 4. (A) The population density in study area and (B) the study area

and PSO-MWNVDs for the studied emergency centers are presented in Figure 5. The optimum values for the parameters of PSO are determined in order to minimize Er for the VDs. The optimum values of $\epsilon_{1}, c_{2}, a$ and population size are selected to be 2, 2, 0.7 and 200, respectively. PSO is reached to the optimal mode of MWNVD in 200 iterations. The changing of mean partial absolute error of PSO-MWNVD in 200 iterations is presented in Figure 6. As shown in Figure 6, the PSO algorithm with a relatively high speed in 12 iterations has achieved value of $6 \%$ error. While the Er error of MWNVD allocation is computed about $37 \%$. The optimum number of ambulances of the Sarem Specialist hospital, Ebne Sina hospital, Fatemeh Zahra health center and Payambaran hospital are obtained 4, 4, 2 and 3 using Eq. 2.

In this study, seventeen wounded with varying level of injuries and the random position were considered. The four above mentioned hospitals must be assigned to these wounded. The process of implementation is explained in the methodology section. The generated MWNVDs and PSO-MWNVDs in Anylogic are shown in Figure 7. The initial VDs that allocated to Sarem Specialist hospital, Ebne Sina hospital, Fatemeh Zahra health center and Payambaran hospital are called "VD,", "VD2", "VD3" and "VD4", respectively.

In the first type of the multi-agent rescue operation, the six wounded are located in $\mathrm{VD}_{1}$, the two wounded are located in $\mathrm{VD}_{2}$, the three wounded are located in $\mathrm{VD}_{3}$ and one wounded is located in $\mathrm{VD}_{4}$. After the delivering help demand messages to

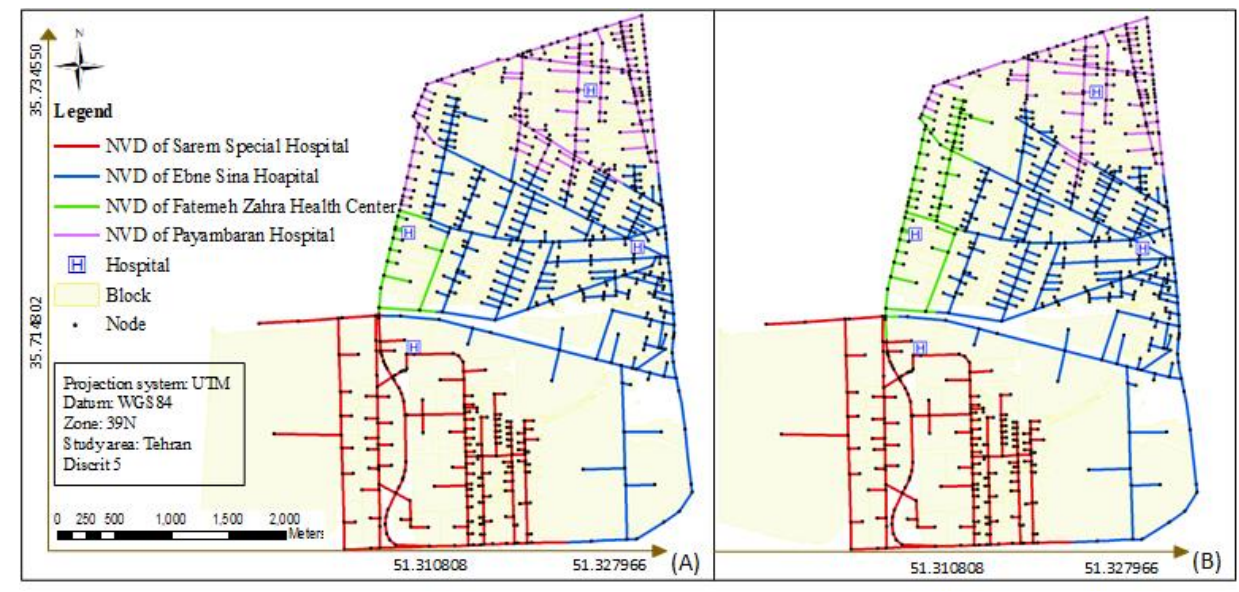

Figure 5. (A) The generated MWNVDs and (B) the generated PSO-MWNVDs

\footnotetext{
${ }^{1}$ Social Security number

${ }^{2}$ Blood oxygen saturation level
} 


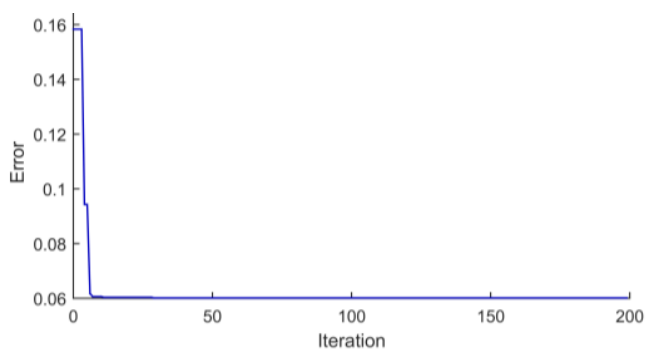

Figure 6. Diagram of the PSO optimization of the MWNVD

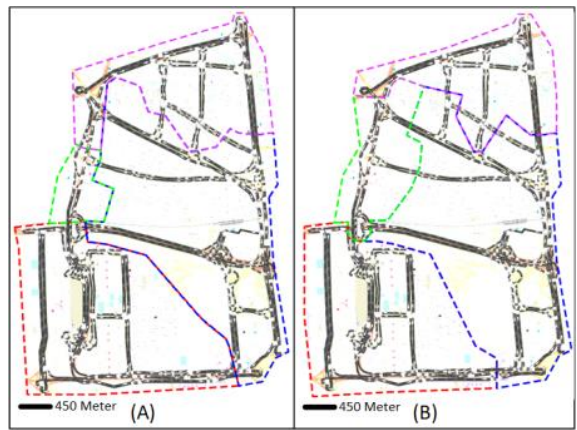

Figure 7. (A) The generated MWNVDs and (B) the generated PSO-MWNVDs in Anylogic

the coordination center agent, the necessary actions are undertaken and the ambulances dispatched to the demand locations according to the generated MWNVDs. In VD $\mathrm{VD}_{1}$ the number of demands is more than the ambulance numbers available in Sarem Specialist hospital. In this condition, the three wounded that have high priority are served first. The other three wounded wait until the ambulances become available again after termination of a mission. When one of the ambulances have undertaken its mission, the high priority of these remained wounded are responded. Also, this trend is continued for the two remained wounded. The assignment process in $\mathrm{VD}_{3}$ is similar to $\mathrm{VD}_{1}$. Due to the fewer wounded than existing ambulances in $\mathrm{VD}_{2}$ and $\mathrm{VD}_{4}$, the wounded people get immediate medical assistance.

In the second type of the multi-agent rescue operation, the location and the priority of the wounded are the same as the first type of the multi-agent rescue operation. Four, two, five and one demands initially are located in $\mathrm{VD}_{1}, \mathrm{VD}_{2}, \mathrm{VD}_{3}$ and $\mathrm{VD}_{4}$ based on PSO-MWNVDs, respectively. Due to the more wounded than ambulances in $\mathrm{VD}_{3}$, the re-allocation processing for Sarem Specialist, Ebne Sina and Payambaran hospitals were performed. After determining the new PSO-MWNVD for these three hospitals (Figure 8 part $\mathrm{C}$ ), the condition of the location of the remained wounded ( 3 wounded) against PSO-MWNVD are determined that are located in the PSO-MWNVD of the Payambaran hospital, Payambaran hospital and Ebne Sina hospital, respectively. So, with this reallocation process, these five wounded are responded faster than the first type of multiagent rescue operation. In $\mathrm{VD}_{1}, \mathrm{VD}_{2}$ and $\mathrm{VD}_{4}$, the ambulances are more than the wounded and the wounded that are located in theses VDs can receive medical assistance without delay. After several minutes, a number of wounded send help demand to the coordination center agent. The assignment process is again performed for the new demands. Figure 9 shows the ambulances and the fire engines actions in Anylogic simulation software.

Mustafa (2013) has shown that if the ambulance sends patient data to the hospital prior his/her arrival, the treatment preparation in the hospital will expedite about two minutes. This results are applied in the implementation of this article.

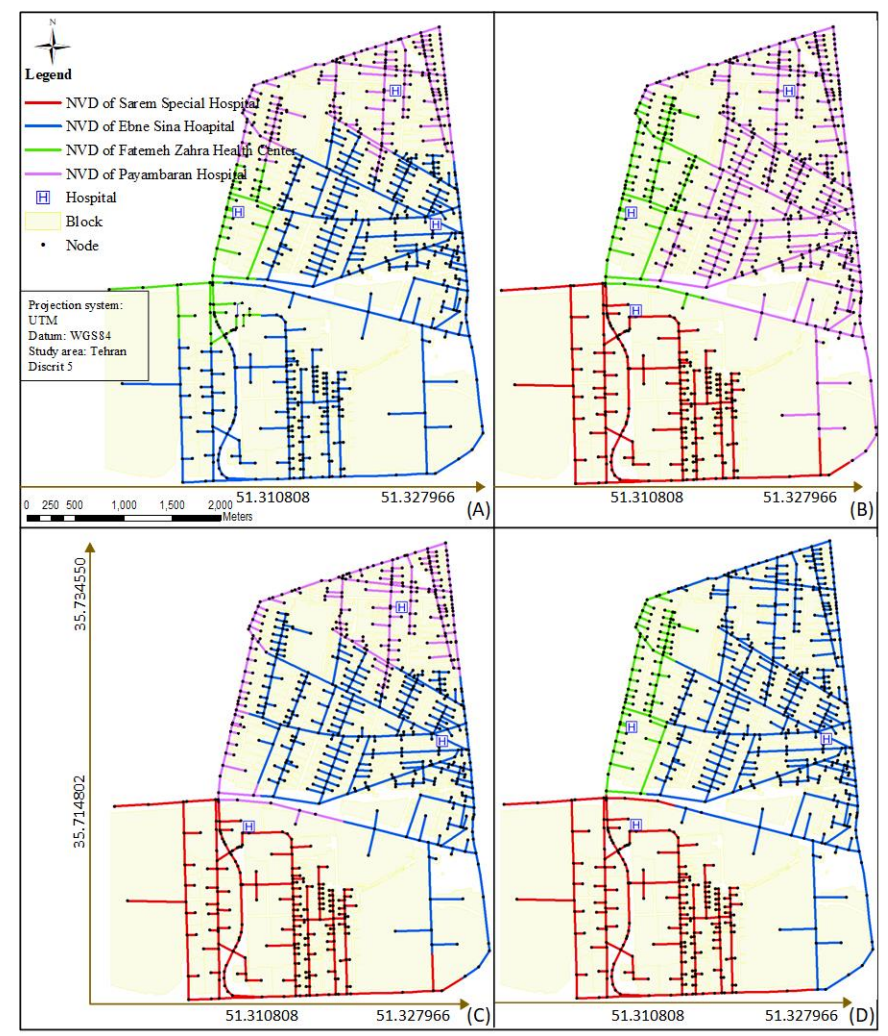

Figure 8. The generated PSO-MWNVD for the emergency centers that have the ambulances without mission when (A) the

ambulances of the Sarem Specialist hospital, (B) the ambulances of the Ebne Sina hospital, (C) the ambulances of the Fatemeh Zahra health center and (D) the ambulances of the Payambaran hospital are not available.

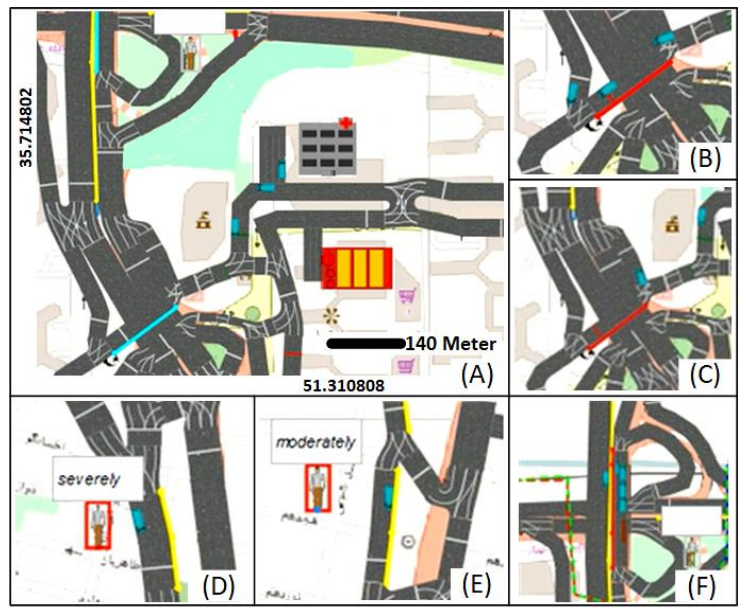

Figure 9. (A) Assignment of the tasks to the ambulances located in the Sarem Special hospital. (B) Catching the ambulance in a blocked street that is displayed in red line. (C) Updating of the

ambulance's route when faced with blocked street. (D)

Reaching ambulance to the wounded locations with severe injury levels. (E) Reaching ambulance to the wounded with moderate injury levels. (F) Resolving the blocked street by the fire engine

The diagram of the arrival times of ambulances to the injured locations are shown in Figure 10. The arrival times of the ambulances for the first and second type of the multi-agent rescue operations are demonstrated in blue stars and red circles, respectively. The average arrival time of the ambulances to the 
injured locations are computed as 11.9 Minutes and 10.6 Minutes in the first and second type of the multi-agent rescue operation, respectively. The results showed that the average arrival time of the ambulances in the second type improved about $10 \%$ in comparison to that of the first type. Also, the diagram of the duration time of the ambulance missions is represented in Figure 11. In the second type of the multi-agent rescue operation, the average ambulance mission time decreased about $17 \%$ compared to that of the first type.

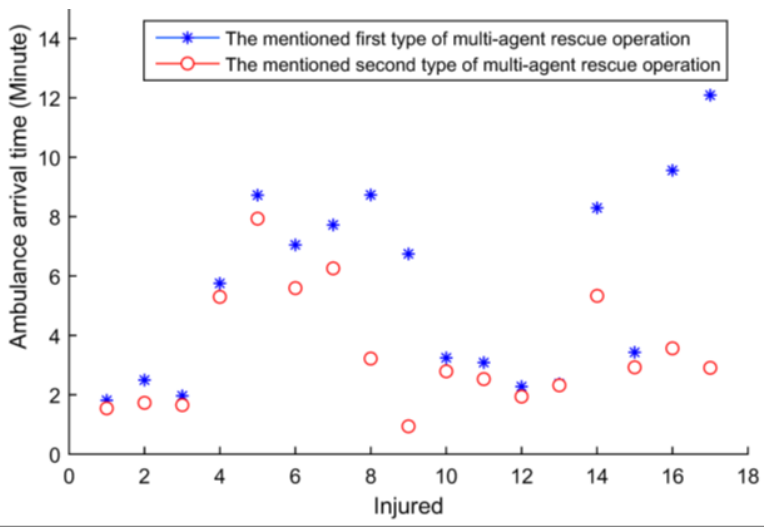

Figure 10. The diagram of arrival time of ambulances to the injured location

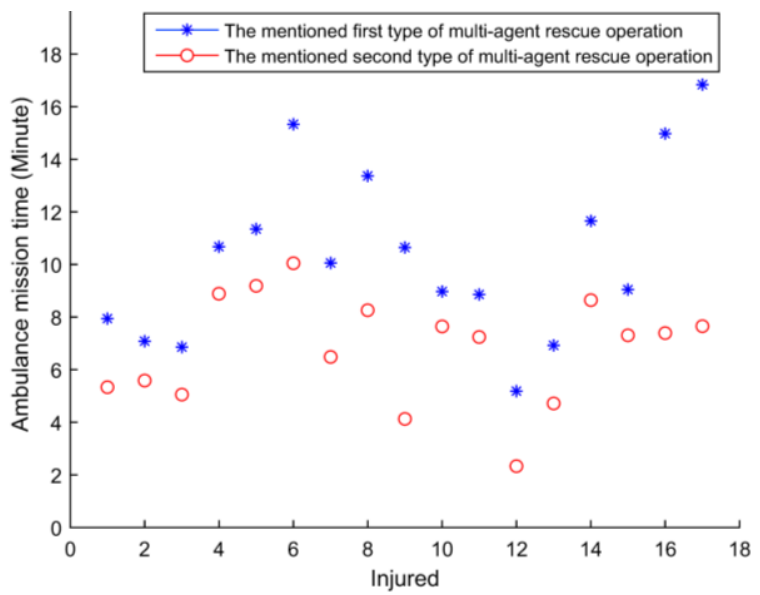

Figure 11. The diagram of the ambulance mission time in order to respond to the injured

\section{CONCLUSION}

In response to the disaster, efficient planning for improving the allocation of medical assistance to emergency victims and wayfinding of the first responders is necessary. The rescue operation time is an important factor in the smart disaster management.

In this study, the multi agent-based model for optimum allocation of the ambulances to the wounded locations according to the urban street network, population density, the number of ambulances exist in emergency centers, optimum wayfinding of emergency vehicles to the injured locations, and the street blockage and smart city facilities to expedite the relief operation is proposed. The proposed rescue operation using the multi agent-based model consists of coordination center, emergency centers, ambulances, fire stations, fire engines and the wounded agents.

After occurring the disaster, the wounded delivered their locations and initial symptoms to the coordination center agent. The rescue operation starts when the location and initial symptoms of the wounded are delivered to the coordination center agent. The patients' injury severity levels are directly relevant to the priority that should be given to each emergency patient for receiving medical assistance. The Bi-label Dijkstra algorithm is used for routing of the ambulances under the objective of minimizing travel length and travel time of ambulances in the street network. The two types of multi-agent rescue operation simulations were implemented and compared. In the first one, the MWNVD allocation for determining the service areas of each emergency center is performed. After specifying the associated MWNVD for each wounded agent, the ambulance is delivered from the emergency center of relevant MWNVD to the wounded agent location. In the case of high demand, if there is an available ambulance located in the emergency center, the allocation is performed, in order to prevent the injured remained helpless.

In the second type of the multi-agent rescue operation, the PSOMWNVD allocation specified the optimum service areas for the emergency centers. The ambulances are able to update their routes in facing with the blocked street. When workload exceeds available ambulance of one or more emergency centers, the PSO-MWNVD allocation is performed again for another emergency centers as far as the new demanded wounded received medical assistance without delay. Also, the information about the condition of the injured people have been transferred to the hospitals prior to an ambulance arrival. In PSO-MWNVD allocation, the partial difference between the estimated and expected population in VDs decreased to $6 \%$. While the mentioned difference computed as $37 \%$ in MWNVD allocation. Also, the average arrival time of the ambulances to the injured location and the average ambulance mission time improved about $10 \%$ to $17 \%$ in the second type of multi-agent rescue operation compared to that of the first type.

Our future work will be focused on more facilities of the smart city for improving the relief operation. Also, considering more agents such as police forces and Red Crescent agents in the multi agent-based model will bring the implementation closer to the reality.

\section{REFERENCES}

Alami-Kamouri, S., Orhanou, G. and Elhajji, S. (2017) Mobile Agent Service Model for Smart Ambulance. Cloud Infrastructures, Services, and IoT Systems for Smart Cities. Springer, 105-111.

Andersson, T. and Värbrand, P. (2007) Decision support tools for ambulance dispatch and relocation. Journal of the Operational Research Society, 58 (2), 195-201.

Bai, Q. (2010) Analysis of particle swarm optimization algorithm. Computer and Information Science, 3, 180.

Bakıc1, T., Almirall, E. and Wareham, J. (2013) A smart city initiative: the case of Barcelona. Journal of the Knowledge Economy, 4, 135-148.

Bandara, D., Mayorga, M. E. and McLay, L. A. (2014) Priority dispatching strategies for EMS systems. Journal of the Operational Research Society, 65, 572-587.

Billhardt, H., Lujak, M., Sánchez-Brunete, V., Fernández, A. and Ossowski, S. (2014) Dynamic coordination of ambulances for emergency medical assistance services. Knowledge-Based Systems, 70, 268-280.

Bostick, N. A., Subbarao, I., Burkle, F. M., Hsu, E. B., Armstrong, J. H. and James, J. J. (2008) Disaster triage systems for large-scale catastrophic events. Disaster medicine and public health preparedness, 2 , S35-S39. 
Chavoshi, S. H., Delavar, M. R., Malek, M. R. and Frank, A. (2008) Landmark-based simulation for agent wayfinding after earthquake.

Eberhart, R. and Kennedy, J. (1995) A new optimizer using particle swarm theory. Micro Machine and Human Science, 1995. MHS'95., Proceedings of the Sixth International Symposium on. IEEE.

Engelbrecht, A. P. (2006) Fundamentals of Computational Swarm Intelligence. John Wiley and Sons.

Engelbrecht, A. P. (2007) Computational Intelligence: an Introduction. John Wiley and Sons.

Fiedrich, F., Gehbauer, F. and Rickers, U. (2000) Optimized resource allocation for emergency response after earthquake disasters. Safety science, $35,41-57$.

Gao, T., Greenspan, D., Welsh, M., Juang, R. R. and Alm, A. (2006) Vital signs monitoring and patient tracking over a wireless network. Proc. IEEE-EMBS 2005. 27th Annual International Conference of the Engineering in Medicine and Biology Society, 2005, 102-105. IEEE.

Ghomian, Z. and Yousefian, S. (2017) Natural Disasters in the Middle-East and North Africa With a Focus on Iran: 1900 to 2015, Health in Emergencies and Disasters Quarterly, 2(2), 53-62.

Gong, Q. and Batta, R. (2007) Allocation and reallocation of ambulances to casualty clusters in a disaster relief operation. IIE Transactions, 39, 27-39.

Haghani, A., Hu, H. and Tian, Q. (2003) An optimization model for real-time emergency vehicle dispatching and routing. The TRB Annual Meeting 2003, 1-23.

Hawe, G. I., Coates, G., Wilson, D. T. and Crouch, R. S. (2015) Agent-based simulation of emergency response to plan the allocation of resources for a hypothetical two-site major incident. Engineering Applications of Artificial Intelligence, 46, 336-345.

Hirokawa, N. and Osaragi, T. (2016) Access time of emergency vehicles under the condition of street blockages after a large earthquake. ISPRS Annals of the Photogrammetry, Remote Sensing and Spatial Information Sciences, 4, 37.

Ibrion, M., Mokhtari, M., Parsizadeh, F. and Nadim, F. (2015) Timescape of the earthquake disasters in Iran: The intricacies of earthquake time and earthquake disaster risk reduction. Geografiska Annaler: Series A, Physical Geography, 97, 197-216.

Imawan, A., Indikawati, F. I., Kwon, J. and Rao, P. (2016) Querying and extracting timeline information from road traffic sensor data. Sensors, 16, 1340.

Karimi, F., Delavar, M. and Mostafavi, M. (2009) Space allocation of educational centers using multiplicatively weighted voronoi diagram. . In $W G$ II/2, II/3, II/4:Workshop on Quality, Scale \& Analysis Aspects of City Models.

Li, D., Shan, J., Shao, Z., Zhou, X. and Yao, Y. (2013) Geomatics for smart cities-concept, key techniques, and applications. Geo-spatial Information Science, 16 (1), 13-24.

Li, X., Zhao, Z., Zhu, X. and Wyatt, T. (2011) Covering models and optimization techniques for emergency response facility location and planning: a review. Mathematical Methods of Operations Research, 74 (3), 281-310.

López, B., Innocenti, B. and Busquets, D. (2008) A multiagent system for coordinating ambulances for emergency medical services. IEEE Intelligent Systems, 23 (5).
Mustafa, A. (2013) Wireless Applications of GIS in Dynamic platforms: a case Study in Laptops and Mobile Phones. PhD Thesis, Mangalore University, India.

Okabe, A., Boots, B. and Sugihara, K. (2000) Spatial Tessellations: Concepts and Applications of Voronoi Diagrams. John Wiley and Sons, Chichester.

Pyrga, E., Schulz, F., Wagner, D. and Zaroliagis, C. (2008) Efficient models for timetable information in public transportation systems. Journal of Experimental Algorithmics (JEA), 12, 2.4.

Reitsma, R., Trubin, S. and Mortensen, E. (2007) Weightproportional space partitioning using adaptive Voronoi diagrams. Geoinformatica, 11 (3), 383-405.

Rini, D. P., Shamsuddin, S. M. and Yuhaniz, S. S. (2011) Particle swarm optimization: technique, system and challenges. International Journal of Computer Applications, 14, 19-26.

Roozemond, D. A. (2001) Using intelligent agents for proactive, real-time urban intersection control. European journal of operational research, 131, 293-301.

Rusell, S. and Norvig, P. (2003) Artificial Intelligent: A Modern Approach. Upper Saddle River: Prentice hall.

Santosa, B. (2006) Tutorial Particle Swarm Optimization. Sukolilo Surabaya: Kampus ITS, 66.

Serafini, P. (1987) Some Considerations About Computational Complexity for Multi Objective Combinatorial Problems. Recent Advances and Historical Development of Vector Optimization. Springer, 222232.

Ticha, H. B., Absi, N., Feillet, D. and Quilliot, A. (2017) A solution method for the Multi-destination Biobjectives Shortest Path Problem. Ecole des Mines de Saint Etienne, CMP, Gardanne, France.

Wang, Y., Colledanchise, M., Marzinotto, A. and Ögren, P. (2014) A distributed convergent solution to the ambulance positioning problem on a streetmap graph. IFAC Proceedings Volumes, 47(3), 9190-9196.

Wang, Z. and Zlatanova, S. (2016) Multi-agent based path planning for first responders among moving obstacles. Computers, Environment and Urban Systems, 56, 4858.

Wood, L. (1974) Spatial interaction and partitions of rural market space. Tijdschrift voor economische en sociale geografie, 65 (1), 23-34.

Yang, X.-S. (2010) Engineering Optimization: An Introduction With Metaheuristic Applications. John Wiley and Sons.

Zhuang, J. and Bier, V. M. (2007) Balancing terrorism and natural disasters-Defensive strategy with endogenous attacker effort. Operations Research, 55 (5), 976-991. 